\title{
Isótopos: los gemelos gordos de los elementos químicos
}

\author{
Fabiola Murguia Flores y \\ Guillermo N. Murray-Tortarolo
}

\begin{abstract}
Resumen
No todos los átomos de un mismo elemento son iguales, algunos son más pesados que otros. Esto se debe a que varían en el número de neutrones que los componen. Así, a los átomos del mismo elemento con diferente masa molecular, debido a variaciones en el número de neutrones, se les conoce como isótopos. Estos parientes "gordos" tienen propiedades muy particulares, que los han vuelto una herramienta de estudio en un sinnúmero de ramas de la ciencia: desde determinar el origen de nuestra luna, el mapeo de la historia climática de la Tierra o la dieta humana, hasta el estudio de las ciencias forenses. En este artículo mostramos cómo el estudio de los isótopos da resultados novedosos e interesantes para la solución de toda clase de preguntas científicas.
\end{abstract}

Palabras clave: isótopos, neutrones, medicina nuclear, dieta humana.

\section{Isotopes: The Fat Twins of Chemical Elements}

\begin{abstract}
Not all atoms of an element are equal, some are heavier than others. This is because they vary in the number of neutrons they contain. Thus, atoms of the same element with different molecular masses, due to variations in the number of neutrons they contain, are known as isotopes. This "fat" relatives have unique physical properties, making them an essential tool in multiple scientific areas: from determining the origins of the moon, mapping the climatic history of Earth or the human diet, to the study of forensic sciences. In this article we show how studying isotopes yields interesting and novel results to solve all sorts of different scientific questions.
\end{abstract}

Key words: isotopes, neutrons, nuclear medicine, human diet.

DOI: http://doi.org/10.22201/codeic.16076079e.2018.v19n5.a5 Recepción: 6/3/2018. Aprobación: 8/8/2018. 


\section{Fabiola Murguia Flores}

fab287@hotmail.com

Es estudiante Doctoral en la Universidad de Bristol en el Reino Unido. Su línea de investigación es el consumo de metano por las bacterias del suelo a nivel global, pero le gustan todos los temas relacionados con los ciclos biogeoquímicos en los suelos. Es además fanática de la divulgación y la enseñanza de la ciencia a todos niveles.

\section{Guillermo N. Murray-Tortarolo}

gmurray@iies.unam.mx

Es Investigador Cátedra CONACyT comisionado al Instituto de Investigaciones en Ecosistemas y Sustentabilidad, Universidad Nacional Autónoma de México. Su tema principal de trabajo es la investigación socio-ecológica para el manejo sustentable de ecosistemas a nivel nacional y global. Le interesa entender los impactos del cambio y la variación climática sobre distintos procesos ecológicos y agronómicos de México y el mundo. También tiene un profundo interés y pasión por la divulgación de la ciencia y por la enseñanza de la misma.

¿Alguna vez has encontrado a alguien idéntico a ti, pero en una ciudad distinta, con distinto peinado, más gordo o más flaco? Y, aun así, te da la sensación de parecerse tanto a ti, que te hace creer que es tu gemelo idéntico. Si existiera..., lo podrías convencer de ir a la escuela o hacer el trabajo pesado por ti, mientras tú juegas videojuegos o ves televisión. Es un sueño que de seguro todos hemos tenido; y, curiosamente, algo así les sucede a todos los elementos químicos: tienen gemelos.

Seguramente has escuchado sobre los isótopos. Y de inmediato los asocias con radioactividad; uno piensa en Chernóbil y Fukushima. Pero los isótopos son nada más y nada menos que los dobles idénticos de los elementos químicos, pero magnificados. Esto significa que los isótopos de un elemento se comportan de manera casi idéntica al elemento químico (hacen el mismo trabajo), pero son más pesados. Son más gordos que el elemento al que pertenecen porque difieren en la cantidad de neutrones que contienen, poseen más, y en esta diferencia de peso existe un gigantesco mundo de información que todos estos pesados nos pueden proporcionar.

Todos los átomos de un mismo elemento tienen la misma cantidad de protones y electrones. Por ejemplo, el hidrógeno contiene un protón y un electrón, el helio dos, y así, sucesivamente, lo que determina su número atómico. Sin embargo, pueden variar en el número de neutrones. Por ejemplo, el hidrógeno tiene tres isótopos: el protio, sin neutrones —éste es el más flaco-; el deuterio, con un neutrón; y, el tritio, con dos (figura 1). Esto confiere propiedades distintas al mismo elemento, como radioactividad y distinto peso atómico. 


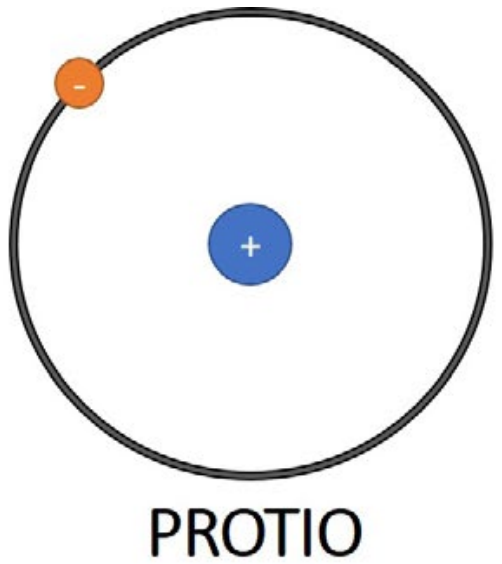

0 neutrones

1 masa atómica

Estable

$99.99 \%$ del $\mathrm{H}$ terrestre

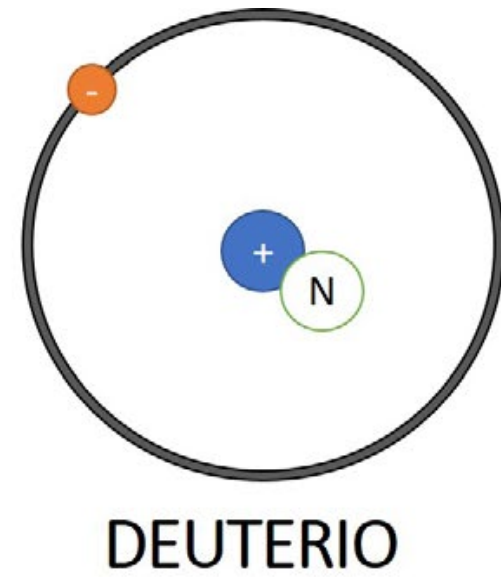

1 neutrón

2 masa atómica

Estable

$0.01 \%$ del $\mathrm{H}$ terrestre

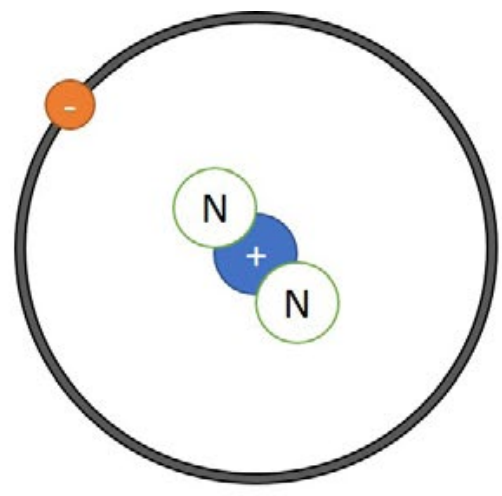

TRITIO

2 neutrones

3 masa atómica

Radioactivo

$0.000001 \%$ del $\mathrm{H}$ terrestre
Figura 1. Isótopos de hidrógeno.
Dicha diferencia en sus propiedades tiene importantes implicaciones en distintas ramas de la ciencia. La manera más sencilla de explicarlo es que los isótopos más pesados (con más neutrones) son más difíciles de mover y, por lo tanto, se almacenan con el tiempo. Se acumulan en los planetas, en las distintas capas de la Tierra, en las diferentes formas de vida e incluso en los seres humanos. Entonces, al estudiar los isótopos obtenemos información sobre una nueva cara del universo que nos rodea y los procesos que lo crearon.

\section{Los isótopos en el universo: el origen de la Luna}

No todas las estrellas ni todos los planetas están hechos de los mismos elementos. Lo más interesante es que aun aquellos que se parecen tienen una composición isotópica única. Esto quiere decir que mediante la medición de distintos isótopos se puede determinar el origen de un planeta o de un asteroide con relación a su sistema solar. El mejor ejemplo de ello ha sido el estudio del origen de la Luna.

Durante mucho tiempo existieron distintas teorías sobre el origen de la Luna. La primera sugería que se trataba de un planetoide y que la gravedad terrestre la capturó. Otra teoría proponía que ambos cuerpos celestes se formaron al mismo tiempo. Una tercera sostenía que una parte de la Tierra explotó y formó la Luna. Y la última, que la Luna se formó tras el choque de un protoplaneta con la Tierra. Las distintas teorías tenían varios puntos a favor y en contra, lo que causó división entre los astrónomos. Pero fue hasta que las misiones Apolo 15 y Apolo 17 llegaron a la Luna y volvieron que finalmente se pudo 


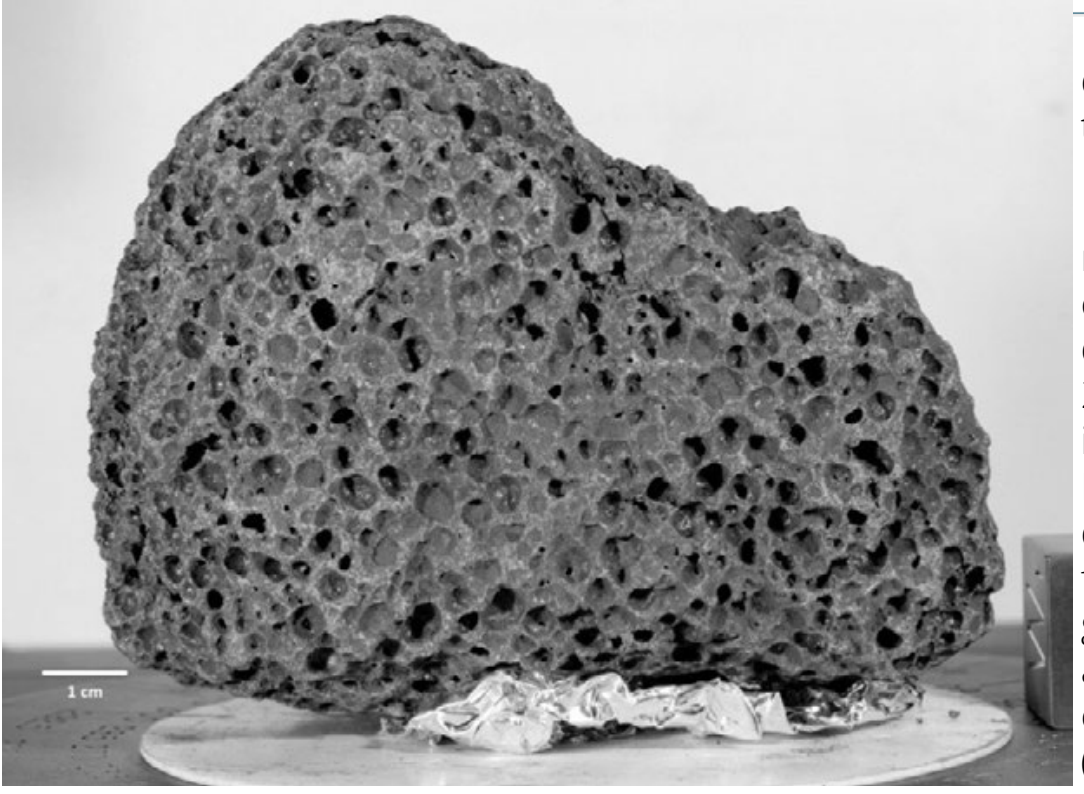

determinar de manera casi contundente cuál de todas ellas era la correcta.

Con la llegada a la Luna se pudieron tomar muestras de rocas de su superficie y analizar su composición isotópica. Distintos estudios publica-

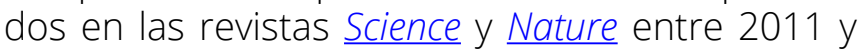
2013 mostraron que la composición relativa de los isótopos de titanio, zinc, selenio y oxígeno de la Luna era igual a la de la Tierra. En otras palabras, que tenían el mismo número de átomos gordos y flacos de cada elemento. Esto implica que el origen de nuestro satélite está forzosamente ligado al origen de nuestro planeta y, lo más probable, es que la teoría del gran impacto sea la correcta (figura 2).
Figura 2.

Fragmento de roca basáltica lunar.

Fuente:

Planetary Geology and Lunar Rocks.

Figura 3.

Isótopos de oxígeno en diferentes tipos de rocas.

Fuente:

modificado de "Oxygen Isotopes and the MoonForming Giant Impact"
Recientemente, Ed Young y colaboradores de ucLA utilizaron un lector de isótopos de alta precisión y lograron determinar que no hay diferencias entre los isótopos de oxígeno en la Tierra y la Luna, lo que los llevó a concluir que el impacto fue causado por un meteorito y el material que salió volando formó el manto de dichos cuerpos celestes. Sus resultados fueron publicados en la revista Science en 2016. El impacto de un cuerpo celeste contra la Tierra en formación también explica la inclinación del eje terrestre y la diferencia en la velocidad de rotación entre la Tierra y la Luna.

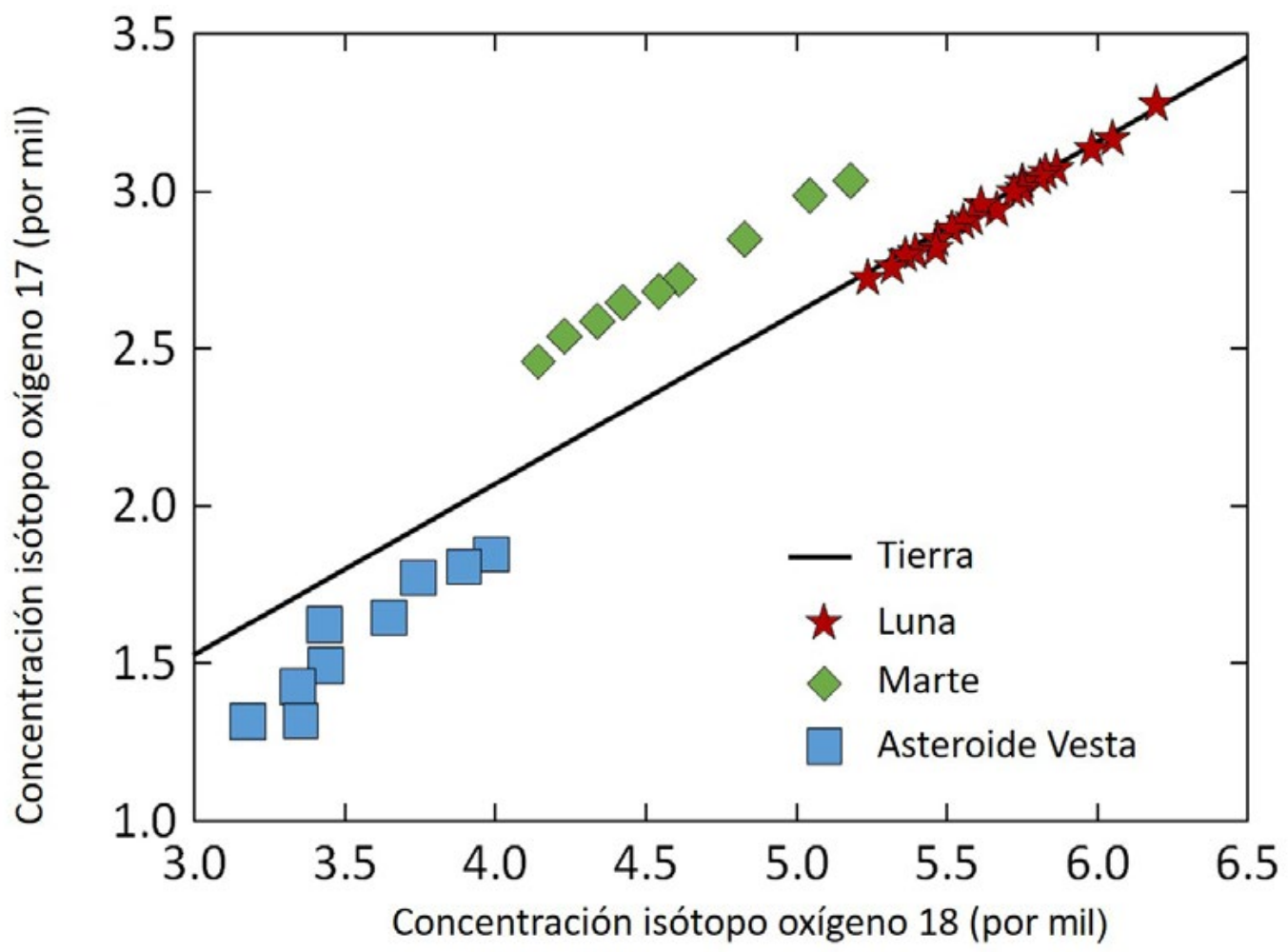




\section{Los isótopos para mapear la historia de la Tierra}

Una huella efectiva con la que contamos para reconstruir la historia de nuestro planeta es la que van dejado los elementos químicos y sus isótopos. Por ejemplo, los hielos de los casquetes polares de la Antártida y Groenlandia han guardado por milenios toda la información de la historia de la atmósfera y el clima de nuestro planeta. En estos lugares la lluvia cae en forma de nieve y nunca se mezcla con su entorno, dando paso a una columna de precipitaciones históricas.

En el corazón de la Antártida, el lugar más frío de la Tierra, con temperaturas de hasta $-90{ }^{\circ} \mathrm{C}$, donde sólo los rusos pudieron construir un centro de investigación, es donde se encuentra la estación de Vostok, y fue allí donde por primera vez se logró perforar el hielo hasta 3623 metros de profundidad. El cilindro de hielo que se obtuvo reveló información sobre los últimos cuatro ciclos glaciales, es decir, el hielo guardaba la historia de 420000 años.
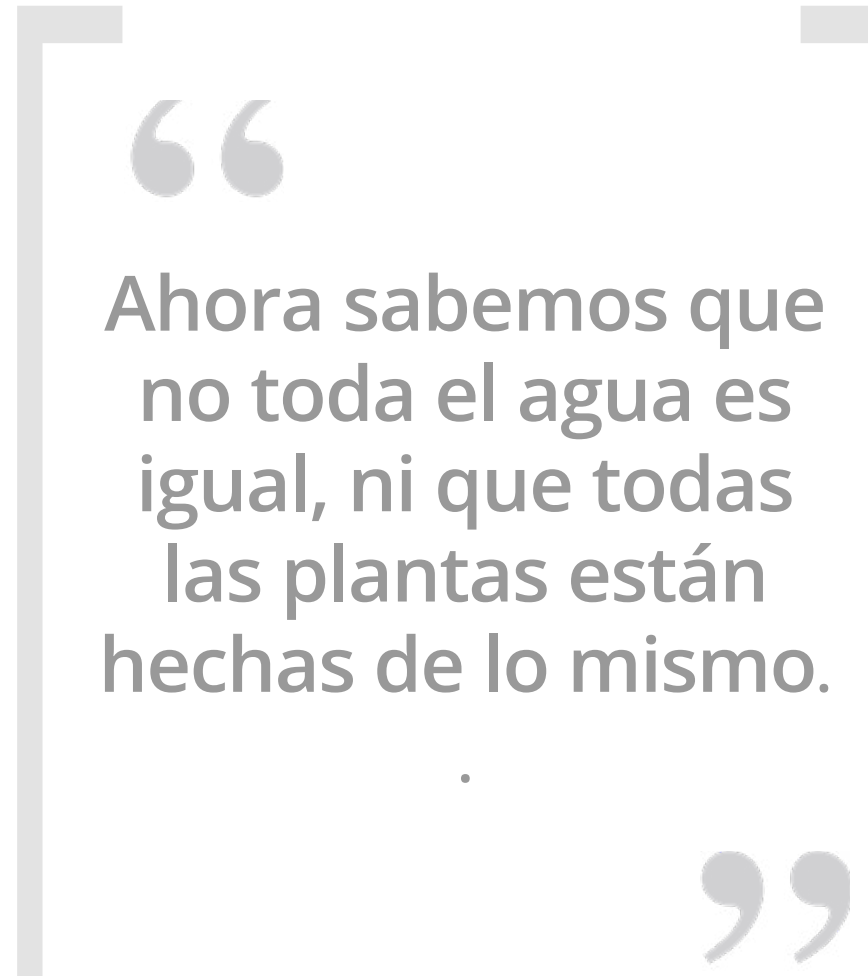

En uno de los artículos más leídos en los últimos tiempos, Jean Robert Petit del laboratorio de glaciología y geofísica ambiental del Centre National de la Recherche Scientifique de Francia y colegas de diversas universidades estadounidenses y rusas pudieron describir el clima y la historia de la atmósfera terrestre de los últimos 420000 años, a partir de estos cilindros. Uno de los análisis consistió en medir los isótopos de oxígeno atrapados en las capas de hielo.

La explicación es que el agua contiene oxígeno 16, pero digamos que también existe un tipo de agua más pesada que contiene un isótopo gordito conocido como oxígeno 18. Esta agua pesada tarda más tiempo en moverse, tiende a evaporarse del mar con mayor dificultad y se adentra menos en los continentes. Estas propiedades aumentan con el frío, pues es más difícil evaporar al oxígeno pesado. Así que la relación entre el oxígeno ligero y el pesado, atrapados en los cilindros de hielo, revela los períodos en los que hubo menor movilidad de agua, principalmente debido a que la temperatura era menor. Usando esta relación como paleo-termómetro se pudieron calcular las temperaturas que había cuando se fueron acumulando las sucesivas capas de nieve. Esto mostró que la temperatura terrestre se había mantenido relativamente estable en todo este tiempo y que un cambio climático como el actual es algo que no se había registrado en el último medio millar de años. 


\section{Los isótopos y la vida}

El registro que ha dejado la vida en el planeta se ha rastreado para reconstruir su historia con ayuda de los fósiles. Sin embargo, no sólo los dinosaurios o los trilobites han dejado su huella en este planeta, también la han dejado las plantas.

Una de las regiones con mayor belleza natural y al mismo tiempo una de las más inhóspitas y salvajes es la sabana africana, donde los pastizales y los arbustos dominan el paisaje. Pero no siempre fue así, en algún tiempo, hace como veinte millones de años, durante el Mioceno, en esa majestuosa pradera reinaban grandes bosques con árboles de diversas especies.

Se puede decir que los pastos y plantas herbáceas son evolutivamente más novedosos y surgieron durante este período. El cambio de vegetación sucedió por cambios en el clima que provocaron sequías al interior del continente, según encontraron David J. Beerling y Colin P. Osborn de la Universidad de Sheffield, Inglaterra.

¿Cómo llegaron a este descubrimiento? Utilizando isótopos, por supuesto. Las plantas utilizan $\mathrm{CO}_{2}$ para realizar la fotosíntesis y ellas pueden discriminar entre $\mathrm{CO}_{2}$ ligero (con carbono 12) y $\mathrm{CO}_{2}$ pesado (con el gordito carbono 13), y prefieren el carbono ligero. Sin embargo, esta discriminación es distinta entre árboles y hierbas, donde esas últimas tienden a acumular una mayor cantidad de isótopos de carbono 13, más pesado. Este elemento, que la vegetación acumula en las hojas, llega finalmente al suelo donde se descompone y deja su huella isotópica. Así, gracias a la conservación y extracción de suelos de hace millones de años se pudo saber que fue durante el Mioceno cuando los grandes bosques abrieron paso a los primeros pastos, que necesitaban menos agua y soportaban más la radiación.

\section{La historia humana a través de los isótopos}

Ahora sabemos que no toda el agua es igual, ni que todas las plantas están hechas de lo mismo. Lo más interesante es que la diferencia entre los isótopos permea a aquellos que se beben el agua o consumen las plantas, incluidos los seres humanos. Entonces, analizando la composición isotópica de fósiles de antiguos humanos e incluso de nuestros ancestros -o de nosotros mismos- se puede entrever el tipo de dieta que éstos tenían y entender más de su estilo de vida.

Por ejemplo, el consumo de frutas, raíces y tubérculos genera marcas isotópicas de carbono diferentes a la que se obtienen al comer cereales o comer pescado. Al analizar distintos dientes del registro fósil humano, Richard Klein de la Universidad de Standford, utilizando isótopos de carbono y nitrógeno, encontró que, en general, los Australopithecus afarensis que vivieron en África hace cuatro millones de años tenían una dieta basada en frutas, más parecida a la 
Figura 4.

Isótopos de los cabellos humanos según el tipo de

dieta.

de los chimpancés actuales. Sin embargo, los primeros Homo (erectus y sapiens) consumían una mayor proporción de granos y carne (35\% de su dieta, hace dos millones de años), misma que aumentó al pasar el tiempo (hasta 80\% hace un millón y medio de años). Esta diferencia en las dietas y la mayor ingesta de proteínas es una de las posibles razones por las cuales nuestro cerebro pudo desarrollarse a mayor velocidad que el de otros primates que sólo se alimentan de frutas.

Otro ejemplo interesante nos lo dan los isótopos del nitrógeno. En general, los peces tienen una composición de dicho elemento distinta a la de los animales terrestres; con lo cual, los seres humanos cuya dieta sea principalmente marina también mostrarán una señal isotópica distintiva. En un artículo, publicado hace casi treinta y cinco años (1983) en la revista Science, Margaret Schoeninger y colaboradores analizaron el registro fósil de los pobladores de América de hace diez mil años. Los resultados mostraron claramente que las civilizaciones de Norteamérica, como los esquimales, dependían fuertemente del consumo de pescado y animales marinos para su supervivencia; al contrario, las culturas más cercanas al Ecuador basaban su alimentación en plantas terrestres.

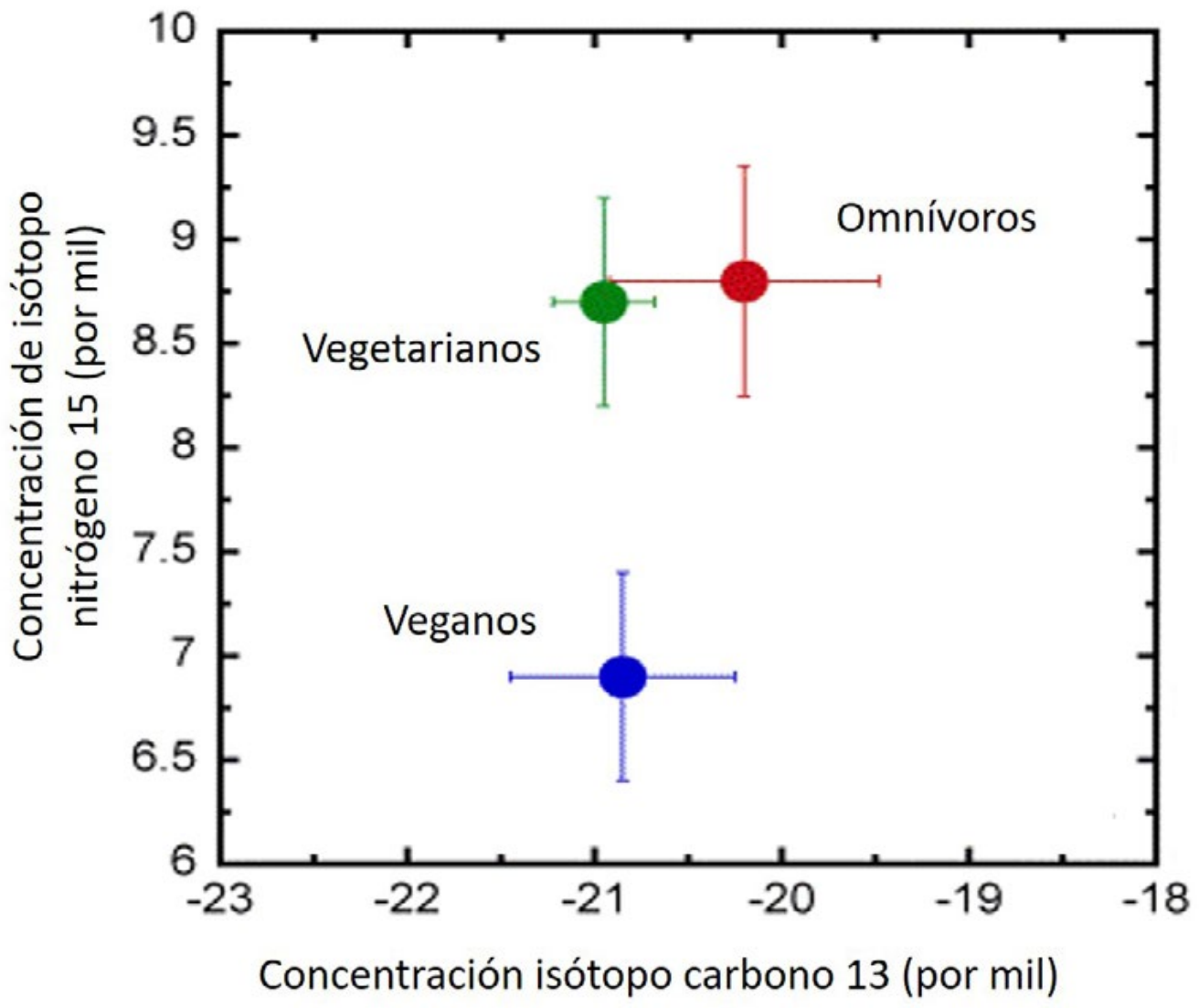

Pero los isótopos también pueden ser utilizados para distinguir entre la dieta de personas aún vivas. Por ejemplo, el cabello de los vegetarianos tiene hasta 3\% menos nitrógeno pesado que el de los que sí comen carne, y el de los 
veganos hasta $6 \%$ menos (ver figura 4). Asimismo, el agua de distintas partes del mundo tiene diferentes concentraciones de selenio pesado, por lo que analizando el cabello de una persona se puede determinar de qué parte del mundo proviene. Esto es particularmente importante en las ciencias forenses, donde el estudio de la composición isotópica de una persona puede proporcionar información relevante de su estilo de vida y origen.

\section{Medicina nuclear}

Pero los isótopos tienen otros usos. Además de ayudarnos a entender eventos históricos, también sirven para detectar y tratar enfermedades. Esta rama de la medicina se conoce como medicina nuclear y tuvo sus orígenes en los últimos cincuenta años. Sus principios se basan en inyectar o absorber de manera oral isótopos radiactivos de algún elemento en particular, por ejemplo, yodo, y mediante el uso de radiografías o tomografías seguir su flujo a través del cuerpo humano. Esto permite detectar de manera fácil y no invasiva la presencia de un sinnúmero de padecimientos como cálculos en la vesícula, problemas cardíacos, infecciones en los huesos y más comúnmente distintos tipos de cáncer. Si bien existen algunos riesgos al inyectar elementos radioactivos en el cuerpo, éstos son en general inocuos y los procedimientos son altamente benéficos para determinar y tratar enfermedades crónicas de manera temprana.

Pero no sólo se utilizan para detectar enfermedades, sino también para tratarlas. Por ejemplo, la administración de yodo radiactivo (yodo 123) es común para el tratamiento de cáncer de tiroides, o los isótopos de samario y estroncio que se utilizan para el tratamiento de cáncer de huesos y de pulmones. Esto tiene cierta ventaja sobre otros métodos de quimioterapia, pues la radiación que se emite es muy localizada, y así se minimizan los efectos secundarios.

\section{Ciencia por descubrir}

Existen otras muchas aplicaciones del estudio de los isótopos; por ejemplo, la determinación del origen del plomo utilizado en balas o explosivos, el estudio del origen de los meteoritos a partir de isótopos de iridio, entre otras. Sin embargo, hay muchos elementos y sus respectivos isótopos que aún no han sido explorados. Por ejemplo, el estaño tiene cerca de diez distintos isótopos por estudiar.

Hay todo un mundo de posibilidades por investigar, quién sabe qué otras maravillas interesantes descubriremos en el futuro a partir de las diferencias en unos pocos neutrones. ¿Quién diría que los gorditos de la naturaleza nos darían tanto de que hablar? 


\section{Referencias}

- Canpu, R. (2012). Forming a Moon with an Earth-like Composition via a Giant Impact. Science, 23(6110), 1052-1055. Dol: https://doi.org/10.1126/science.1226073.

* Elkins-Tanton, L. (2013). Planetary science: Occam's origin of the Moon. Nature Geosciences, 6, 996-998. Dol: https://doi.org/10.1038/ngeo2026.

* Young, E. D., Kohl, I. E., Warren, P. H., Rubie, D. C., Jacobson, S. A. y Morbidelli, A. (2016). Oxygen isotopic evidence for vigorous mixing during the Moon-forming giant impact. Science, 351(6272), 493-496. Dol: https://doi.org/10.1126/science. aad0525.

* Petit, J. R., Jouzel, J., Raynaud, D., Barkov, N. I., Barnola, J. M., Basile, I. et al. (1999). Climate and atmospheric history of the past 420,000 years from the Vostok ice core, Antarctica. Nature, 399(6735), 429. Dol: https://doi.org/10.1038/20859.

* Beerling, D.J.y Osborne, C. P. (2006). The origin of the savanna biome. Global change biology, 12(11), 2023-2031. Dol: https://doi.org/10.1111/j.1365-2486.2006.01239.x.

* Klein, R. G. (2013). Stable carbon isotopes and human evolution. Proceedings of the National Academy of Sciences, 110(26), 10470-10472. Dol: https://doi.org/10.1073/ pnas.1307308110.

* Schoeninger, M. J., DeNiro, M. J. y Tauber, H. (1983). Stable nitrogen isotope ratios of bone collagen reflect marine and terrestrial components of prehistoric human diet. Science, 220(4604), 1381-1383. Dol: https://doi.org/10.1126/science.6344217.

\section{Cómo citar este artículo}

* Murguia Flores, Fabiola y Murray-Tortarolo, Guillermo N. (2018). Isótopos: Ios gemelos gordos de los elementos químicos. Revista Digital Universitaria (RDU). Vol. 19, núm. 5 septiembre-octubre. DOI: http://doi.org/10.22201/codeic.16076079e.2018. v19n5.a5. 